\title{
Agotamiento Emocional en estudiantes universitarios del área de la salud
}

\author{
Emotional exhaustion in university students within the health field
}

Doris Amparo Barreto-Osma1* orcid.org/0000-0002-4542-0944

Henry Augusto Salazar-Blanco' ${ }^{1}$ orcid.org/0000-0003-2135-5006

1. Universidad Autónoma de Bucaramanga

Fecha de recepción: Junio 27 - 2019

Fecha de revisión: Agosto 07- 2019

Fecha de aceptación: Diciembre 29 - 2020

Barreto-Osma DA, Salazar Blanco HA. Agotamiento Emocional en estudiantes universitarios del área de la salud. Univ. Salud. 2021;23(1):3039. DOI: https://doi.org/10.22267/rus.212301.211

\section{Resumen}

Introducción: El Agotamiento Emocional (AE) es la disminución de energía, sentimiento de desgaste emocional y físico, asociados a una sensación de frustración y fracaso. Los estudiantes universitarios experimentan situaciones que conjugan diversos elementos estresores. Objetivo: Describir el AE en estudiantes universitarios del área de la salud en instituciones de educación superior (IES) de la ciudad de Bucaramanga - Colombia. Materiales y métodos: Estudio cualitativo que siguió un diseño flexible de tipo descriptivo interpretativo, articulado al análisis de contenido. Se realizaron 175 entrevistas abiertas de forma consentida y voluntaria. Resultados: Se encontraron tres categorías con sus respectivas subcategorías: agotamiento emocional (consecuencias fisiológicas y psicológicas), dificultades asociadas (académicas, socio-familiares), estrategias de afrontamiento (activas y resolutivas, pasivas y negativas). Conclusiones: Los estudiantes experimentan el $\mathrm{AE}$, hecho que repercute en su salud física y mental, disminución del desempeño académico y afectación en la vida familiar y social. Las estrategias de afrontamiento para algunos de ellos, son de carácter resolutivo, mientras que para otros son de tipo negativo. El AE puede ser mitigado con estrategias de prevención y apoyo psicosocial.

Palabras clave: Agotamiento emocional; síntomas afectivos; estrategias de afrontamiento; estudiante. (Fuente: DeCS, Bireme).

\begin{abstract}
Introduction: Emotional exhaustion (EE) is a state of emotional and physical burnout and energy depletion that are associated with feelings of frustration and failure. University students experience situations that combine various stressful elements. Objective: To describe EE health field students from universities of the city of Bucaramanga - Colombia. Materials and methods: A qualitative study with a flexible, descriptive and interpretative design was carried out, which included 175 open interviews conducted in a consensual and voluntary manner. Results: Three categories and respective subcategories were revealed: (i) emotional exhaustion (physiological and psychological consequences); (ii) associated difficulties (academic, social and familiar); and (iii) coping strategies (active and decisive or passive and negative). Conclusions: Students experience EE, which affects their physical and mental health, decreases their academic performance and disturbs their familiar and social interactions. Some students apply decisive coping strategies, while others use negative ones. EE can be mitigated through prevention strategies and psychosocial support.
\end{abstract}

Keywords: Emotional exhaustion; affective symptoms; coping strategies; student. (Source: DeCS, Bireme).

\footnotetext{
*Autor de correspondencia:

Doris Barreto

e-mail: dbarreto@unab.edu.co
} 


\section{Introducción}

El ser humano a lo largo de su ciclo vital se enfrenta a diferentes ámbitos de tipo académico, laboral, familiar y social, para enfrentarlos se requieren constantes cambios; para ello es necesario de la adquisición y actualización de conocimientos y habilidades, aspectos que requieren del empleo de distintos recursos de afrontamiento para disminuir el impacto en la salud de las personas(1).

Para suplir las necesidades del mundo globalizado y competitivo, las personas deciden estudiar y preparase a través de la educación superior, lo que trae consigo retos académicos muy diversos, pero también representa uno de los puntos más altos de estrés, que van desde las grandes cargas de trabajo, los procesos de ajuste a la vida universitaria y las alteraciones significativas del entorno familiar y social $(2,3)$.

Los requerimientos a los que están expuestos los estudiantes, les genera desgaste mental y físico, relacionado con la sobrecarga de tareas, falta de tiempo para realizar los trabajos académicos y preparar las evaluaciones(4). Así pues, los sujetos se enfrentan a situaciones posiblemente amenazadoras, que pueden llegar a sobrepasar sus propias posibilidades, empezar a sentir niveles de estrés, aumentar la predisposición a estados de depresión y ansiedad(5), afectando su rendimiento académico(6). También, experimentan fases de agotamiento(7) y disminución en los elementos de respuesta, perdiendo su interés por las actividades académicas ${ }^{(8)}$, sintiéndose inútiles ante cualquier esfuerzo, lo que lleva a perfilarlos a un fenómeno denominado síndrome de Burnout $(9,10)$.

Según la Organización Mundial de la Salud (OMS), el síndrome de Burnout es un problema en ambientes académicos y laborales e impacta a distintas personas, en especial a aquellos que tienen contacto permanente con otros, lo que representa alta entrega y respuesta inmediata; si no se tienen los recursos necesarios, puede llegar a presentarse ansiedad, frustración, baja autoestima, soledad, depresión y otros problemas de índole emocional ${ }^{(11,12) .}$

Este síndrome llamado también "estar quemado", desde el enfoque psicosocial se ha estructurado en tres componentes: la baja realización personal, la despersonalización y el agotamiento emocional(13). El primer componente se expresa por la tendencia a evaluarse negativamente, emergiendo cuadros de ansiedad, alterando la concentración, el rendimiento en el estudio u otros escenarios y la insatisfacción en todos los resultados obtenidos. El segundo, la despersonalización, se manifiesta por sentimientos negativos, actitudes de cinismo, irritabilidad y culpabilización hacia los otros. El tercer componente, agotamiento emocional (AE), se convierte en el eje central de dicho síndrome, manifestado por la fatiga emocional, física y el total agotamiento energético y de recursos emocionales(13). Del mismo modo, el AE es la disminución de los recursos afectivos que producen la sensación de no poder aportar y dar más y se manifiesta con síntomas somáticos y psicológicos ${ }^{(14)}$.

También es visto como la pérdida del gusto y la calma en la ejecución de las actividades cotidianas propias del sujeto, (estudio, trabajo, vida social, entre otras)(15). Se podría decir entonces que corresponde a una disminución de las facultades para relacionarse adecuadamente con los demás, la persona se siente agobiada tanto física como psicológicamente, con ineficacia y desesperanza no pudiendo dar más de sí misma (16,17). Por estas razones el $\mathrm{AE}$, se convierte en la respuesta más destacada a las situaciones estresantes del entorno y es el indicador más frecuente en la etapa temprana del Burnout.

El AE afecta a estudiantes universitarios, dado las frecuentes experiencias estresantes, los nuevos entornos académicos, cambios en la metodología de enseñanza-aprendizaje, alteraciones significativas del entorno socio - familiar (separación de amigos y familiares, el traslado a una nueva ciudad), así como a variables de tipo individual relacionadas con el nivel de preparación, la falta de una fuente de financiación para su semestre, asumir otros roles de tipo laboral o ser padres a temprana edad(18). Esto permite explicar en su conjunto una mayor vulnerabilidad en los estudiantes universitarios a padecer períodos de cansancio emocional sostenido ${ }^{(19) .}$

Si bien es cierto, que todos los universitarios se encuentran expuestos a estas situaciones, distintos estudios han observado que los cuadros de $\mathrm{AE}$, son más frecuentes en carreras de la salud(20,21). Las principales fuentes se relacionan con eventos y largas jornadas académicas teórico-prácticas (propia 
del proceso de aprendizaje), la presión de los docentes, la realización de procedimientos y decisiones esenciales que repercuten en el bienestar de otros, así como, asuntos de tipo psicosocial; esto hace que se encuentren sometidos a un ambiente emocionalmente extenuante(22,23), se sientan sobreexigidos, carentes de recursos psicológicos y físicos, sin ninguna fuente de reposición, con ausencia de energía para enfrentar las actividades $(24,25)$, de modo tal, que tendrán dificultades para una mejor adaptación $(17,26)$.

Las consecuencias del $\mathrm{AE}$, son mediatizadas y reguladas por un proceso llamado afrontamiento, visto como un asunto dinámico, en respuesta a exigencias objetivas y a valoraciones subjetivas que viven a diario las personas(27). El afrontamiento es "el conjunto de esfuerzos cognitivos y a manejar (minimizar, reducir, dominar o tolerar) las solicitudes internas y externas de una determinada situación agobiante"(28).

La identificación de estrategias de afrontamiento empleadas por los universitarios es de total pertinencia, ya que tiene implicaciones relevantes desde lo educativo(28) y lo clínico. Las estrategias empleadas ya sean funcionales, productivas o negativas tienen estrecha relación con la calidad de vida, el bienestar, la adaptación y ajuste en el ámbito universitario(29,30). La presente investigación enfatiza en la dimensión del Agotamiento Emocional como respuesta básica al estrés y por tal razón se planteó como objetivo describir el $\mathrm{AE}$ en estudiantes universitarios del área de la salud en instituciones de educación superior (IES) de la ciudad de Bucaramanga - Colombia.

\section{Materiales y métodos}

\section{Diseño}

El estudio con enfoque cualitativo se enmarcó dentro de un diseño flexible de tipo descriptivo interpretativo, articulado a las estrategias de análisis de contenido temático, como herramienta que permite sistematizar los datos obtenidos de los participantes. Se optó por el análisis categorial cualitativo, en el que se relacionan "el contexto del texto con el contexto social". A partir de la transcripción de las entrevistas se descompone el texto en unidades con significado propio, para luego agruparlas en categorías semejantes según criterios establecidos en los objetivos de la investigación(31).

\section{Participantes}

La población objeto de estudio fueron estudiantes del área de la salud de cuatro universidades de la ciudad de Bucaramanga. Se realizó un muestreo no probabilístico por conveniencia, resaltando que la aceptación de cada participante fue voluntaria y éticamente intrínseca. Dado el enfoque cualitativo del estudio, los investigadores contactaron a 175 participantes bajo criterios de costo - efectividad y disponibilidad de la muestra.

\section{Instrumento}

Se realizó una guía de entrevista que constó de 10 preguntas abiertas construidas a partir la revisión teórica(17-19), relacionada con el síndrome de Burnout y su principal componente el AE. Dichas preguntas fueron validadas por un grupo de tres expertos (un profesional de bienestar universitario, un psicólogo clínico y un especialista en seguridad y salud en el trabajo) que las calificaron siguiendo criterios de suficiencia, claridad, coherencia, relevancia e inocuidad. Los jueces aprobaron la estructura del instrumento y sugirieron ajustes en la redacción de algunos términos. El instrumento permitió la flexibilidad y obtener información relacionada con los diferentes puntos de vista de los participantes y el modo en que se han subjetivado las experiencias de estudiar carreras relacionadas con el área de la salud.

\section{Procedimiento}

Para desarrollar la investigación se siguieron cuatro fases. En la primera se realizó la presentación de la investigación a los grupos de interés en las diferentes universidades. En la segunda, los voluntarios decidieron participar firmando consentimiento informado y se procedió a realizar las entrevistas por parte de los dos investigadores con experiencia en técnicas cualitativas. En la tercera se hizo una transcripción de las narrativas de los estudiantes con un análisis previo para obtener las categorías emergentes. En la cuarta, una vez obtenida la información, de acuerdo al método de contenido temático fue posible integrar herramientas analíticas: el software de análisis de datos cualitativos QSR NVivo10 que permitió facilitar el proceso de organización de la información, codificación, identificación y estructuración de categorías de tipo emergente. Y a través del análisis de contenido como herramienta para sistematizar la información mediante la interpretación de los datos obtenidos(31,32), tomando 
como premisa el rigor de la objetividad y elementos de la subjetividad de los participantes. Se optó por el análisis categorial cualitativo, el cual tiene dos principios fundamentales, por un lado, se tiene en cuenta el contexto del texto, es decir, el material transcrito producto de las entrevistas $\mathrm{y}$, por otro lado, la experiencia y comprensión de la realidad.

En este orden de ideas, un análisis de contenido categorial parte de datos textuales, descompone el texto en unidades, luego se agrupan en categorías, siguiendo los principios de la analogía, refiriendo las semejanzas y similitudes que existan en función de los criterios previamente establecidos según los objetivos de la investigación( ${ }^{(33)}$. Cabe señalar que los resultados de la investigación no pretenden ser generalizados, sino que se constituyen en un aporte para la comprensión y el significado de la realidad psicosocial, expresada en el agotamiento emocional de los jóvenes universitarios del área de la salud.

\section{Consideraciones éticas}

Según la Resolución 8430 de 1993 del Ministerio de Salud por la cual se establecen las normas científicas, técnicas y administrativas para la investigación en salud, este estudio se clasifica en la categoría "riesgo mínimo". La Investigación contó con el aval ético de la Universidad Autónoma de Bucaramanga, todos los entrevistados colaboraron de manera voluntaria y se les dio la oportunidad de aceptar o rechazar su participación en el estudio. Todos los sujetos fueron mayores de edad, diligenciaron y firmaron un formato de consentimiento informado.

\section{Resultados}

Participaron del estudio 175 universitarios de los programas de psicología $(44,6 \%)$, enfermería (32\%) y medicina $(23,4 \%)$ de diferentes semestres de cuatro IES de la ciudad de Bucaramanga- Colombia. De éstos el 62,8\% corresponde al sexo femenino y el $37,2 \%$ fue del género masculino. Las edades de los participantes estuvieron en los rangos de 18 a 25 años (59\%), entre 26 a 30 años (34,2\%) y 30 a 35 o más años (6,8\%). El estado civil se distribuyó en solteros $(72,5 \%)$, casados $(17,1 \%)$ y unión libre $(10,4 \%)$. Los estratos socioeconómicos fueron estrato $2(14,9 \%)$, estrato $3(44,6 \%)$, estrato 4 $(28 \%)$ y estrato $5(12,5 \%)$ (Tabla 1$)$.

Las categorías emergieron a partir de las distintas percepciones de los estudiantes del área de la salud, se partió de su propia realidad social en toda su complejidad construida en las distintas acciones y se dio una aproximación a su comprensión.

Tabla 1. Características de los universitarios

\begin{tabular}{|c|c|c|c|c|c|c|c|c|c|c|c|c|}
\hline \multirow{2}{*}{ Carrera Universitaria } & \multicolumn{10}{|c|}{ Semestre } & \multicolumn{2}{|c|}{ Total } \\
\hline & I & II & III & IV & $\mathbf{V}$ & VI & VII & VIII & IX & $\mathbf{X}$ & $\mathbf{F}$ & $\%$ \\
\hline Psicología & & 11 & 9 & 10 & 13 & 9 & 10 & 8 & 8 & & 78 & 44,6 \\
\hline Enfermería & & 9 & 12 & 8 & 9 & 11 & 7 & & & & 56 & 32,0 \\
\hline Medicina & & 12 & 8 & 9 & 6 & 6 & & & & & 41 & 23,4 \\
\hline \multicolumn{13}{|l|}{ Género } \\
\hline Femenino & & & & & & & & & & & 110 & 62,8 \\
\hline Masculino & & & & & & & & & & & 65 & 37,2 \\
\hline \multicolumn{13}{|l|}{ Edad (años) } \\
\hline $18-25$ & & & & & & & & & & & 103 & 59,0 \\
\hline $26-30$ & & & & & & & & & & & 60 & 34,2 \\
\hline 30-35 o más & & & & & & & & & & & 12 & 6,8 \\
\hline \multicolumn{13}{|l|}{ Estado Civil } \\
\hline Solteros & & & & & & & & & & & 127 & 72,5 \\
\hline Casados & & & & & & & & & & & 30 & 17,1 \\
\hline Unión Libre & & & & & & & & & & & 18 & 10,4 \\
\hline \multicolumn{13}{|l|}{ Estrato socioeconómico } \\
\hline Estrato 2 & & & & & & & & & & & 26 & 14,9 \\
\hline Estrato 3 & & & & & & & & & & & 78 & 44,6 \\
\hline Estrato 4 & & & & & & & & & & & 49 & 28,0 \\
\hline Estrato 5 & & & & & & & & & & & 22 & 12,5 \\
\hline Total entrevistados & & & & & & & & & & & 175 & 100 \\
\hline
\end{tabular}


La presentación de la información se organizó tomando cada una de las categorías emergentes y sus respectivas subcategorías, las cuales surgieron de las entrevistas, fueron segmentadas en unidades de análisis, siendo el insumo para la codificación (Tabla 2). Se levantaron tres categorías de primer orden (agotamiento emocional, dificultades asociadas y estrategias de afrontamiento), dentro de las cuales se interpretó el contenido general con fragmentos textuales enunciados por los estudiantes (E).

Tabla 2. Categorías y subcategorías de análisis

\begin{tabular}{lc}
\hline \multicolumn{1}{c}{ Categoría y subcategorías de análisis } & Frecuencia \\
\hline Agotamiento Emocional & \\
Consecuencias fisiológicas & 747 \\
Agotamiento Físico & \\
$\quad$ Consecuencias Psicológicas & 628 \\
$\quad$ Fatiga mental & 594 \\
$\quad$ Fatiga emocional & 638 \\
Dificultades asociadas & 448 \\
$\quad$ Académicas & \\
$\quad$ Socio-familiares & 402 \\
Estrategias de afrontamiento & 378 \\
$\quad$ Activas y resolutivas & \\
$\quad$ Pasivas y negativas &
\end{tabular}

Los códigos se analizaron por contenido temático y la frecuencia de éstos son la suma total de las palabras literales y/o relacionadas, a partir de las expresiones de los participantes. Las dimensiones centrales para efectos del presente estudio son: La primera, agotamiento emocional, con la subcategoría consecuencias fisiológicas, referida como la respuesta producida por las demandas corporales, los esfuerzos prolongados en las actividades relacionadas con el estudio, así como las respuestas neuroendocrinas y asociadas al sistema nervioso autónomo como aumento de presión sanguínea, incremento de la actividad estomacal e intestinal, dolor de cabeza entre otros(27). La subcategoría, consecuencias psicológicas, vista como la exposición intelectual continua y la tensión producida por las exigencias emocionales que abarcan las sensaciones subjetivas de alteración emocional como la ansiedad, el temor, la excitación, el miedo, la ira y la depresión(25).

La segunda categoría, son las dificultades asociadas, con las subcategorías académicas y socio-familiares.
La tercera y última categoría, está relacionada con las estrategias de afrontamiento.

\section{Agotamiento emocional}

Sentimiento de agotamiento, que experimentan los estudiantes del área de la salud a causa de los compromisos académicos, que derivan posteriormente en una actitud negativa, bajo compromiso con sus actividades, creencias de poca capacidad en lo que realizan. Las manifestaciones se dan a nivel físico y psicológico(26).

Dentro de las consecuencias físicas, que son frecuentes e incómodas, está el agotamiento en los estudiantes, quienes refieren sentirse cansados, con fatiga física, decaídos, sensación de pesadez; todo ello derivado de horarios extenuantes, de sobreesfuerzos y requerimientos académicos. Así lo narró ET5:"sentirme cansando y fatigado la mayor parte del tiempo, agotado y hacer esfuerzos para atender a clase y comprender las distintas temáticas". Los anteriores aspectos se manifiestan en la mayoría de los participantes por sintomatologías relacionados con dolor corporal, tensión y espasmos musculares principalmente en la parte alta de la espalda y el cuello "el dolor en el cuello y la espalda en ocasiones es insoportable"(E24), también presentan problemas digestivos, migrañas frecuentes y alteración del sueño, "tengo problemas digestivos, pues el tiempo es un factor que me juega malas pasadas y no alcanzo a alimentarme bien, $\mathrm{mi}$ gastritis es fuerte y me genera dolores" (E89) "cansancio constante, duermo muy poco y eso me produce dolor de cabeza, mal genio y desgaste" (E131).

A nivel de las consecuencias psicológicas está la fatiga mental y la emocional. La primera es originada por los distintos compromisos académicos de los estudiantes, esto les demanda un gran esfuerzo mental de forma continua, involucrando la comprensión, el razonamiento, la memoria; aspectos que al no regularse les ocasiona falta de atención, errores en sus actividades y disminución en la habilidad para solucionar problemas, “... últimamente he experimentado agotamiento mental, olvido de algunas actividades, fechas importantes $y$ cometo errores principalmente en los parciales" (E126). La segunda, la fatiga emocional, considerada como el cansancio o agotamiento ante la respuesta del estrés a situaciones de difícil manejo y como un mecanismo de defensa extremo que la mente pone 
en marcha cuando no puede asimilar los momentos experimentados(7), suele venir asociado con sentimientos de angustia, depresión y minusvalía. Los participantes refieren inquietud, nerviosismo, cierta inseguridad, así lo manifestó E23 “.... a veces he experimentado mucha angustia, ansiedad, estrés continuo e impotencia de no realizar todas las actividades", hay en ellos también alteración en el estado de ánimo, irritabilidad o falta de autocontrol, "mi estado de ánimo ha estado con altibajos, a veces ganas de llorar, pero también ira, rabia y poco control de mis emociones..." (E102). Además, experimentan preocupación por la parte financiera, "me angustia que no consiga el dinero para el siguiente semestre, eso es crítico" (E111).

Al analizar las características sociodemográficas de los universitarios del área de la salud, en relación con la categoría de agotamiento emocional se encontró que el género femenino tiene mayor frecuencia de consecuencias psicológicas y fisiológicas: "Ha sido pesado, debido a que no dispongo de todo el tiempo que quisiera...en ocasiones me he enfermado porque tengo jornadas muy extensas y poder cumplir con mi familia y estudio" (E15). Respecto al estado civil, los participantes casados, mostraron más indicadores de fatiga mental y emocional que los estudiantes solteros, siendo importante resaltar que la situación se acentúa en aquellos estudiantes que además de pareja tienen hijos menores de edad "... cuando se tiene en mi caso un hogar con hijos y esposo, trabajar y responder, estudiar cien por ciento en todo, es un poco complicado, a veces estresante..." (E13).

\section{Dificultades Asociadas}

Las dificultades asociadas, se refieren a otros efectos colaterales que presentan los estudiantes con $\mathrm{AE}$, pues se reconoce que los compromisos académicos conllevan a una disminución de las interacciones en la vida familiar y social.

A nivel académico los participantes refieren falta de tiempo para los estudios, pues no cuentan con la organización y los espacios necesarios para responder a la sobrecarga de tareas escolares (ensayos, trabajos de investigación) y a la preparación de exámenes(34,35), Así lo comentó E69 "Escaso tiempo para poder desarrollar tareas y dedicarle tiempo a profundizar en las materias y dar buenos resultados en los parciales", lo que conlleva a un bajo desempeño educativo; ratificando así el esfuerzo que les demanda cumplir con el estudio y otros roles que asumen, reflejado en el "bajo rendimiento que estoy teniendo en materias que necesitan toda mi concentración y atención"(E104). También frente a estos hechos, los estudiantes pueden dilatar la terminación de su carrera y cada vez se torna más difícil culminarla o algunos piensan abandonarla, lo que se convierte en un gran desafío "a veces he pensado dejar la carrera, pues soy madre soltera y me queda muy difícil" (E123).

Desde el punto de vista socio - familiar, se identifican barreras de tiempo para compartir con la familia, "...tener poco tiempo para disfrutar en familia es especial con mi hija de 4 años" (ET47), también presentan una significativa reducción de la vida social, el limitado disfrute e integración con compañeros y amigos, escasa participación en actividades recreativas, de ocio y culturales; aspectos importantes en la etapa en que se encuentran estas personas "Me es muy complicado disfrutar tiempo con mi familia y amigos durante los periodos académicos (E113). Lo anterior, dificulta la construcción de una red social de apoyo, siendo esto un factor de riesgo para era población.

\section{Estrategias de Afrontamiento}

La última categoría, estrategias de afrontamiento, son tomadas como los procesos concretos y las acciones que se utilizan en cada situación problemática para obtener resultados multidireccionales según diversos factores o variables(34). Los estudiantes universitarios del área de la salud afrontan de diversas maneras las múltiples situaciones que enfrentan. Algunos tienen afrontamientos más activos y resolutivos, que implican una reinterpretación positiva del proceso o vivencias que están teniendo. Una de estas estrategias es la construcción y utilización de redes sociales "... a pesar del poco tiempo que tengo, termino buscando a mis amigos o familiares para sentirme apoyado por ellos" (E37), es así como el soporte social puede disminuir el efecto de ciertos momentos estresantes y ser amortiguador de los eventos negativos. De igual modo, están encaminados a solucionar las problemáticas que asumen, aprendiendo procedimientos nuevos de afrontamiento "cuando tengo un problema que me está causando malestar, busco solucionarlo, me tomo un tiempo, lo analizo y busco estrategias" (E97). Otras alternativas usadas son por ejemplo el humor "Considero que soy un tipo que tengo buen sentido del 
humor y molesto con todos mis compañeros, eso me distrae y me cambia el chip" (E95). Hay también grupos de estudiantes que afrontan los momentos de estrés con estrategias poco favorables como es evitación del problema, descontrol en sus emociones, reacciones adversas contra sí mismos o sus entornos, consumo de sustancias y automedicación. Se expresaron frases como estas: "No es nada fácil, afrontar y poder manejar mis roles, estudiante, trabajo los fines de semana, madre soltera y muchas veces me descontrolo" (E14), "Cuando estoy rendida y sin fuerzas tomo energizantes y cuando el dolor o el malestar aparece tomo analgésicos... si me automedico y a veces pastas para dormir, tomo de todo usted me entiende" (E28), "Cuando las cosas se me salen de las manos termino no yendo a estudiar, me busco incapacidades o pago supletorios "(E159).

\section{Discusión}

El estudiante universitario se convierte en la razón fundamental del proceso educativo, en la medida que se va formando, se convierte en generador de conocimiento. Por esa razón, la información pertinente y el continuo monitoreo de las situaciones particulares, tales como: vivencias, experiencias, formas y modos de aprender; permitirá a la universidad conocer más de cerca a esta población y formar profesionales integrales, con excelencia académica.

Si bien, las investigaciones en esta área han logrado cuantificar los niveles de AE, es necesario conocer y comprender las experiencias y significados que tienen las personas frente a este fenómeno. Situaciones como la fatiga, la disminución de recursos afectivos y la falta de energía están vinculadas a características subjetivas, situacionales, contextuales y culturales ${ }^{(22,23)}$. Para citar un ejemplo, las alteraciones de los calendarios académicos universitarios en las jornadas de paro influyen de manera diferente en los estudiantes de acuerdo a sus propias ideologías y responsabilidades, mientras que para algunos puede ser un tiempo de descanso o de lucha por los ideales de justicia social, para otros representa gastos y dilación en su título profesional.

De acuerdo con los resultados arrojados por la presente investigación, el $\mathrm{AE}$ es una problemática presente en los estudiantes de psicología, enfermería y medicina, con una afectación de su salud física y mental. Esta situación concuerda con los estudios realizados por autores que resaltan la alta incidencia del síndrome de Burnout en estudiantes de ciencias de la salud(20).

Según los hallazgos de este proyecto, los estudiantes del área de la salud, presentan $\mathrm{AE}$ cuyo punto de partida parece ser el desbalance entre las obligaciones, los recursos que tienen los participantes y la carencia de estrategias para afrontar de forma adecuada las múltiples exigencias de las instituciones educativas. Esto corrobora lo planteado por Gómez y Gudín(5), quienes resaltan el impacto del rendimiento académico, pues las alteraciones de la salud ocurren principalmente en evaluaciones parciales y exámenes finales.

El AE tiene dos consecuencias muy marcadas, las físicas y las psicológicas. Las primeras están asociadas a síntomas somáticos y se caracterizan por presentar mayor susceptibilidad a la enfermedad. La segunda puede expresarse por actitudes negativas sobre sí mismo, sentimientos de inferioridad, baja productividad, sensaciones de incompetencia, sentimientos de depresión, cansancio emocional, que al no ser controlados puede conllevar a ideas de suicidio $\mathrm{u}$ otras alteraciones $\mathrm{y}$ finalmente la exacerbación de algún tipo de trastorno mental(17).

Los estudiantes, presentan un alto nivel de síntomas somáticos reflejados en alteraciones de tipo osteomuscular, afecciones gástricas, cefaleas y alteraciones en el patrón de sueño, resultado del cansancio, agotamiento y la fatiga que experimentan en los periodos académicos. Desde el aspecto psicológico los jóvenes expresan abiertamente sentir estrés la mayor parte del tiempo, asociado a sentimientos negativos e intranquilidad, angustia, tensión, descontrol emocional y falta de concentración; siendo probable que fracasen en diversas tareas y esto vaya retroalimentando las emociones negativas. Vale la pena discutir sobre la formación de los estudiantes en el autocuidado de su propia salud, pues en muy pocos casos se evidencia la búsqueda de ayuda profesional máxime cuando ellos se están formando para atender la salud de la población. De esta manera se resalta la importancia de programas de prevención y de intervención en los niveles leves y medios del AE, para minimizar los daños, tal como lo señalan Escudero, Colorado y Palacio(17). 
Considerando que la respuesta al $\mathrm{AE}$ depende de la disposición personal para hacer frente a los eventos y a la propia situación $(7,8)$, el estudio mostró que no todos los universitarios del área de la salud lo padecen de la misma forma, esto depende de la percepción que tenga cada uno de ellos y los recursos para afrontarlos, dichos recursos encontrados en las experiencias de los estudiantes entrevistados se pueden explicar como una forma de autoeficacia que contribuye a generar un estado mental positivo que aumenta la adaptación y el logro académico.

A nivel socio- familiar, los estudiantes, experimentan disminución de la vida social, espacios insuficientes para compartir con los amigos y con los miembros de su núcleo familiar. Como lo expresaron los universitarios, muchas interacciones se pierden o se establecen solo a nivel de redes sociales, sin alcanzar a ser un mecanismo de apoyo social. En este sentido, Ávila et al.(3), resaltan que el apoyo social, actúa como un potenciador en el logro y el buen desempeño de los estudiantes, ya que contribuye a que afronten los estresores propios de su ambiente con mayores probabilidades de éxito.

Usualmente, el afrontamiento permite identificar elementos que intervienen en la relación dada entre los niveles de $\mathrm{AE}$ y los síntomas de enfermedad; dicha relación es recíproca, las acciones que hace una persona para desafiar un problema afectan la valoración del mismo y el subsecuente del afrontamiento. Las estrategias utilizadas por las personas, son consideradas como catalizadoras de la perturbación emocional; éstas pueden ser favorables como por ejemplo búsqueda de apoyo social, solución de problemas, regulación emocional, y, por tanto, no se dará el malestar. Igualmente, es importante señalar que existen respuestas que buscan controlar el agotamiento una vez que éste ha aparecido, con actividades relacionadas con el ocio(30). En caso opuesto, podría verse afectada la salud de forma negativa, así como también los estilos de vida, llevándolos al deterioro en los hábitos alimenticios, aumento en el consumo de algunas sustancias como el tabaco, el alcohol, la cafeína, los energizantes, la automedicación, así como el sedentarismo y el deterioro de la apariencia personal(3).

Al indagar sobre las estrategias de afrontamiento que utilizan los estudiantes ante las distintas situaciones, algunos presentan estilos positivos centrados en la solución de problemas, búsqueda de apoyo, participación en grupos sociales, sentido del humor, diversión (televisión, cine) y deporte (gimnasio, trotar, yoga). Las personas con bajo bienestar afrontan las circunstancias de forma negativa o reactiva, evitando el problema o aliviando la tensión a través del uso de diferentes sustancias, aspectos que aceleran el deterioro de la calidad de vida. Como lo señala Rodríguez(29), las estrategias de afrontamiento para los estudiantes, deben orientarse desde edades tempranas a promover el esfuerzo, la constancia, la diversión y la autoeficacia con el fin de proteger a los universitarios de los estresores académicos.

\section{Conclusiones}

Los estudiantes del área de la salud presentan $\mathrm{AE}$ caracterizado por consecuencias tanto fisiológicas como psicológicas evidenciadas en agotamiento físico, fatiga mental y emocional. Dichas situaciones de agotamiento se exacerban por las exigencias académicas y los problemas socio-familiares a los que se enfrentan.

Lo anterior supone, plantear desde las universidades y los departamentos de bienestar universitario programas complementarios de salud física y apoyo psicológico, para dotar a los estudiantes del área de la salud de recursos y estrategias de afrontamiento, orientadas a disminuir el nivel de AE y así lograr una mayor calidad de vida de los jóvenes que se esfuerzan por alcanzar un proyecto de vida profesional. Por tanto, se requieren medidas a nivel formativo que permitan prevenir el desarrollo del síndrome y de sus consecuencias sobre el bienestar psicológico de éstos.

A partir de la descripción del $\mathrm{AE}$ en universitarios del área de la salud, surgen nuevas perspectivas de investigación en la que se busque relacionar esta problemática con situaciones propias del ambiente educativo tales como las clases teóricas, el estilo de los docentes, y los escenarios complejos del ámbito hospitalario (enfermedades terminales, epidemias), o del contexto social (poblaciones vulnerables, zonas de conflicto). Así mismo, se recomienda realizar investigaciones que permitan comparar el $\mathrm{AE}$ de estudiantes del área de la salud con otras áreas como humanidades e ingenierías. 
Conflicto de intereses: Los autores no declaran conflictos de intereses.

\section{Referencias}

1. García Retana J. Modelo educativo basado en competencias: importancia y necesidad. Revista Electrónica "Actualidades Investigativas en Educación" [Internet]. 2011;11(3):1-24. Recuperado https://www.redalyc.org/articulo.oa?id=44722178014

2. Herrara S. Análisis de la percepción del mercado laboral de jóvenes estudiantes universitarios. EducacionUdcEs [Internet]. 2009;448-57. Disponible en: http://www.educacion.udc.es/grupos/gipdae/congreso/Xc ongreso/pdfs/t2/t2c26.pdf

3. Castillo Ávila I, Barrios Cantillo A, Alvis Estrada AR. Estrés académico en estudiantes de enfermería de Cartagena, Colombia. Acad Stress Nurs Students Cart Colomb [Internet]. 2018;20(2):1-N.PAG. Disponible en: https://revistas.javeriana.edu.co/index.php/imagenydesarr ollo/article/view/16457

4. González Cabanach R, Fernández Cervantes R, González Doniz L, Freire Rodríguez C. Estresores académicos percibidos por estudiantes universitarios de ciencias de la salud. Fisioterapia. 2010;32(4):151-8.

5. Gómez CMV, Gundín OA. Estresores académicos percibidos por estudiantes universitarios y su relación con el burnout y el rendimiento académicos. Anuario de psicología. [internet]. 2016;46(2):90-97. Disponible en: https://www.sciencedirect.com/science/article/pii/S0066 512616300125

6. Alcalá-Pacas A, Ocaña-Sánchez MA, Rivera-Arroyo G, Almanza-Muñoz JDJ. Síndrome de Burnout y rendimiento académico en una escuela de medicina. Neurol Neurocir y Psiquiatr. 2010;43(1-4):13-25.

7. Domínguez-Lara SA. Agotamiento emocional académico en estudiantes universitarios: ¿Cuánto influyen las estrategias cognitivas de regulación emocional? Educación Médica. [Internet] 2018;19(2):96-103. Disponible en: https://www.sciencedirect.com/science/article/pii/S1575 181316301607

8. Casuso M. Estudio del estrés, engagement y rendimiento académico en estudiantes universitarios de Ciencias de la Salud. [internet]. 2011. Disponible en: https://riuma.uma.es/xmlui/bitstream/handle/10630/492 6/TD\%20Maria\%20Jesus\%20Casuso\%20Holgado.pdf?sequ ence $=1$

9. Serrano T, Salguero J, Ayala J, García M, Meza J, Mejía C. Síndrome de Burnout en estudiantes de seis facultades de medicina de Colombia, estudio metacéntrico. CIMEL. [internet]. 2016;21(2):29-34. Disponible en: https://www.cimel.felsocem.net/index.php/CIMEL/article/ view/642

10. Merino-Soto $\mathrm{C}$, Fernández-Arata M. Ítem único de burnout en estudiantes de educación superior: estudio de validez de contenido. Educación Médica. [internet]. 2017;18(3):195198. Disponible

en: https://www.sciencedirect.com/science/article/pii/S1575 181316300924
11. Saborío Morales L, Hidalgo Murillo LF. Revisión bibliográfica Síndrome de Burnout. Med Leg Costa Rica Edición Virtual. 2015;32(1).

12. Rosales Ricardo Y, Rosales Paneque F. Burnout estudiantil universitario. Conceptualización y estudio. Salud Ment [Internet]. 2013;36(4):337-45. Disponible en: http://www.medigraphic.com/cgibin/new/resumen.cgi?IDARTICULO=44895

13. Vizoso-Gómez C, Arias-Gundín O. Resiliencia, optimismo y burnout académico en estudiantes universitarios. Eur J Educ Psychol. 2018;11(1):47

14. Lledó-Carreres A, Perandones-González TM, Herrera-Torres L, Lorenzo G. Cansancio emocional, autoestima y satisfacción con los estudios en estudiantes universitarios. [internet]. 2014. Disponible en: https://rua.ua.es/dspace/bitstream/10045/63189/1/2014 _Lledo_etal_INFADRevPsicologia.pdf

15. Tomaschewski-Barlem JG, Lunardi VL, Lunardi GL, Barlem ELD, Da Silveira RS, Vidal DAS. Burnout syndrome among undergraduate nursing students at a public university. Rev Lat Am Enfermagem. 2014;22(6):934-41.

16. Novoa-Sandoval P, Burgos-Muñoz RM, Zentner-Guevara JA, Toro-Huamanchumo CJ. Burnout syndrome in nursing students from a Peruvian university. Inventory MB. 2007;2017-9. Disponible en: http://dx.doi.org/10.1016/j.riem.2017.05.001

17. Marenco Escuderos A, Suárez Colorado Y, Palacio Sañudo J. Burnout académico y síntomas relacionados con problemas de salud mental en universitarios colombianos. Psychologia. 2017;11(2):45.

18. García-Ros R, Pérez-González F, Pérez-Blasco J, Natividad LA. Evaluación del estrés académico en estudiantes de nueva incorporación a la universidad [Academic Stress Evaluation in New Students Joining University]. Rev Latinoam Psicol. 2012;44(2):143-54.

19. Torres LH, Mohand LM, Espinosa SC. Cansancio emocional en estudiantes universitarios. DEDiCA. Revista de Educação e Humanidades. [internet]. 2016;(9):173-191. Disponible en:

https://dialnet.unirioja.es/servlet/articulo? codigo $=542938$ 1

20. Martos Á, Pérez-Fuentes M del C, Molero M del M, Gázquez JJ, Simón M del M, Barragán AB. Burnout y engagement en estudiantes de Ciencias de la Salud. Eur J Investig Heal Psychol Educ. 2018;8(1):23.

21. Martos Á, del Carmen Pérez-Fuentes M, del Mar Molero M, Gázquez JJ, del Mar Simón, M, Barragán AB. Burnout y engagement en estudiantes de Ciencias de la Salud. European Journal of Investigation in Health, Psychology and Education. [internet]. 2018;8(1):23-36. Disponible en: https://www.formacionasunivep.com/ejihpe/index.php/jo urnal/article/view/223

22. Ferrel Ortega FR, Ferrel Ballesta L, Cantillo Aguirre AA, Jaramillo Campo J, Jim SM. Variables académicas y sociodemográficas relacionadas con el Síndrome de Burnout, en estudiantes de Ingenierías y Ciencias de la Salud de una universidad estatal de Colombia. Psicogente [Internet]. 5 de junio de 2017 [citado 13 de enero de 2020];20(38). Disponible en: http://revistas.unisimon.edu.co/index.php/psicogente/arti cle/view/2555

23. Garza M del R, Sanmiguel MF, Muñoz A, Rodríguez CE. El estrés en estudiantes de medicina al inicio y final de su 
formación académica. Rev Iberoam Educ. 2015;1(66):10522.

24. Florenzano R. Salud mental y características de personalidad de los estudiantes universitarios en Chile. Calidad en la Educ. 2005;(23):105.

25. Uribe T MÁ, Illesca P M. Burnout en estudiantes de enfermería de una universidad privada. Investig en Educ Médica [Internet]. 2017;6(24):234-41. Disponible en: http://dx.doi.org/10.1016/j.riem.2016.11.005

26. González-Ramírez MT, Landero-Hernández R, Tapia-Vargas A. Percepción de salud, cansancio emocional y síntomas psicosomáticos en estudiantes universitarios. Ansiedad y estrés. [internet]. 2007;13(1).

27. Lemos M, Henao-Pérez M, Lopez-Medina D. Estrés y Salud Mental en Estudiantes de Medicina: Relación con Afrontamiento y Actividades Extracurriculares. IMedPub Journals. 2018;24(2:3):1-8.

28. Quiñones ÁSÁ, Mogollón GJM, Arenas DJ, Díaz JPB. Estilos y estrategias de afrontamiento y rendimiento académico: una revisión empírica. Enfoques. 2014;1(1):15.

29. Morales Rodríguez FM. Estrategias de afrontamiento en una muestra de estudiantes universitarios. Int J Dev Educ Psychol Rev INFAD Psicol. 2018;2(1):289.

30. Farkas C. Estrés y afrontamiento en estudiantes universitarios. Psykhe. [Internet]. 2011;11(1). Disponible en:

http://www.psykhe.cl/index.php/psykhe/article/view/456

31. Díaz Herrera C. Investigación cualitativa y análisis de contenido temático. Orientación intelectual de revista Universum. RGID [Internet]. 2018;28(1):119-42. Disponible en:

https://revistas.ucm.es/index.php/RGID/article/view/608 13

32. Hernández Sampieri Roberto, Fernández Collado Carlos, Baptista Lucio Pilar. Metodología De La Investigación: $6 a$. ed. --. México D.F.: McGraw-Hill, 2014.

33. Aguirre-García JC, Jaramillo-Echeverri JC. Aportes Del Método Fenomenológico a La Investigación Educativa. Rev Latinoam Estud Educ. 2012;8(2):51-74.

34. Casari L, Anglada J, Daher C. Estrategias de aprendizaje y ansiedad ante los exámenes en estudiantes universitarios. Rev Psicol Pontif Univ Católica Argentina, [Internet]. 2014;32(2):243-69. Disponible en: http://dialnet.unirioja.es/servlet/extart?codigo=3091343

35. Carrillo Regalado S, Ríos Almodóvar J, Ríos Almodovar J. Trabajo y rendimiento escolar de los estudiantes universitarios. El caso de la Universidad de Guadalajara, México. Rev la Educ Super. 2013;42(166):9-34. 